\title{
Giving According to Preferences
}

\section{Decision-making in the Group Dictator Game}

\author{
By Axel Franzen and Sonja Pointner*
}

\begin{abstract}
We study the decision process in a group dictator game in which three subjects can distribute an initial endowment between themselves and a group of recipients. The experiment consists of two stages: first, individuals play a standard dictator game. Second, individuals are randomly matched into groups of three and communicate via instant messaging regarding the decision in the group dictator game. In contrast to former studies our results show that group decisions do not differ from individual decisions in the dictator game. Furthermore, the analysis of the chat history reveals that players make proposals according to their preferences as revealed in the single dictator game and that these proposals in groups drive the final allocation.
\end{abstract}

\section{Introduction}

The standard approach in game theory and economics is to analyze individual decision-making. However, in real-life settings many decisions are not taken by lone actors but in groups such as committees, company boards, policy councils, juries or households. In a recent review of the experimental literature comparing group decisions with individuals' decisions, Charness and Sutter (2012) conclude that groups generally make better self-interested decisions. This is particularly true when the performance of groups is compared to the performance of individuals in problem-solving tasks. One of the most prominent examples is the beauty-contest game in which individuals (or groups) are asked to estimate a certain proportion (e.g. two-thirds) of the average number (e.g. of the interval $[0,100])$ chosen by other players. In the beauty-contest game the equilibrium choice is zero and groups generally get closer to the equilibrium solution than do individual players (e.g. Kocher / Sutter 2005; Sutter 2005). Similar findings apply to other problem-solving games such as the ,urn experiment“ (e.g. Blinder / Morgan 2005; Charness / Karni / Levin 2007) and the „letter-to-number" task (Laughlin / Bonner / Miner 2002).

In decision situations where the Nash-equilibrium strategy is at odds with the Pareto-efficient outcome, groups more often follow the Nash equilibrium strategy. This finding refers particularly to experiments using the Prisoners' Dilemma game (e.g. Insko et al. 1990). Similarly, Charness, Rigotti, and Rustichini (2007) find that individuals follow the payoffdominant strategy in the Prisoners' Dilemma more closely if they make the decision on behalf of a group (with ,payoff communality“). Gillet, Schram, and Sonnemans (2009) compare group and individual decision-making in a common resource-pool game. They find that groups are more competitive than individuals, both depleting the pool sooner and overharvesting more often. Similarly, groups are less trusting than individuals in the trust game, transferring a lower amount to the second player (Kugler et al. 2007), and they end the game sooner in the centipede game (Bornstein / Kugler / Ziegelmeyer 2004). Hence, in social dilemmas groups make the emergence of cooperation less likely.

In this paper, we are interested in whether groups rather than individuals also behave more selfishly in fairness games, a question which is not addressed in the review of Charness and Sutter (2012). Compared to the evidence accumulated in problem-solving tasks or social dilemma games the existing results are much less clear with respect to fairness games.

* We would like to thank Marc Keuschnigg, Tobias Wolbring, and the reviewers for many helpful comments on an earlier version of this manuscript. 
Fairness decisions are often studied using the dictator game (Kahneman / Knetsch / Thaler 1986). Numerous studies have shown that individual dictators give substantial amounts (e.g. 20 to $30 \%$ ) of the received endowment to the recipient (Camerer 2003; Engel 2011). However, there are only two studies so far comparing groups and individuals in the dictator game and they each come up with opposing results. Cason and Mui (1997) report an altruistic shift in groups as compared to individuals in the dictator game, while Luhan, Kocher, and Sutter (2009) report a selfish shift. ${ }^{1}$ We extend this prior research by taking a detailed look into the decision dynamics of the groups. Our subjects first played the simple dictator game individually (stage 1), and thereafter played in groups of three players (stage 2). In this second stage the group members communicate via instant messages about the distributive choice. Our results suggest that the three-player groups allocate on average the same amount to others as do the three group members when taking the decision in isolation. Moreover, a detailed analysis of the chat protocols suggests that the groups' final allocation depends on the communication process. Groups that exchanged many selfish arguments (suggesting keeping the whole endowment) kept more for themselves as compared to groups that exchanged more moderate or equal-split proposals. Moreover, the number of selfish or fair proposals exchanged depends on the groups' composition in terms of selfish or fair players. Thus, our results suggest, contrary to the two prior studies, that the allocation given by groups in the dictator game depends simply on the type of players that make up a group. Hence, there is neither an altruistic shift nor a selfish shift.

The remainder of the paper is organized into four sections: Section two discusses the reasons that groups might make different decisions than individuals in the dictator game. Here we draw on evidence from experimental economics and the social psychological research on groups and discuss how the findings apply to decision-making in the group dictator game (GDG). In section three we describe the experimental procedure and section four reports the findings. Finally, section five concludes with a summary and a discussion of the results.

\section{Theory and hypotheses}

In problem-solving tasks, the advantage of groups over individuals is quite obvious. First, groups have more resources (more brains) and the chances of a group containing a member who discovers the solution are higher as compared to a single individual. This argument also applies to social dilemma games in which the Nash-equilibrium strategy might not be obvious. Second, groups have the advantage that members can discuss the problem. This encourages thinking harder about a problem and enables the easier discovery of errors. Charness and Sutter (2012) also point out that through discussion, groups are better able to put themselves into the minds of the other (out-group) co-players. Therefore, groups are better in anticipating the other party's strategy and consequently are also better in finding the best reply strategy. Thus, in the beauty-contest game, groups progress more often to higher levels in the reasoning process. This argument implies that groups are more than the sum of their members and should perform better than the best player contained in the group. ${ }^{2}$

Since the dictator game does not involve an intellectual problem, having more brains in a group is of no help in this game. Hence, the social preferences of the individuals of the group should determine a group's decision, e.g. a majority of selfish individuals should push

1 There is a third study by Dufwenberg and Muren (2006) using the group dictator game (also called team dictator game). However, their study is concerned with effects of gender composition of the group and not with the difference between individual and group decision-making which is our focus.

2 The first argument states that groups outperform the average individual. This is also referred to as „weak synergy“. The second argument states that groups outperform even the best group member. This criterion is also termed as ,strong synergy effect“ (Larson 2010). 
the group towards a selfish distribution while a majority of generous players should induce a more altruistic outcome. Therefore, groups should decide according to the preferences of their members. Contrary to strategic games, the possibility of discussing the situation should also not be of much help in the dictator game. Since the recipient cannot influence the result, a group discussion cannot improve anticipation of others' behavior and it is not possible for the groups to eliminate any errors in the allocation decisions.

However, research in Social Psychology has often revealed that group behavior differs from individuals' behavior in motivational aspects. Most importantly, groups are empirically often found to be more competitive than individuals (e.g. Insko et al. 1990; Sutter / Kocher / Strauss 2009; Wildschut et al. 2003). Individuals tend to identify with their in-group and draw a stronger distinction between themselves and out-group members (Tajfel 1978). This identification with a group often results in a shift towards more competitive behavior towards out-group members.

Furthermore, decision-making in groups implies that the behavior of individuals is more visible, at least by the other group members. This observability of behavior in groups might affect groups in different ways. On one side much research has demonstrated that individuals adhere more strongly to social norms and show more socially desirable behavior when they feel observed. Research with the simple dictator game suggests that individuals donate money to recipients not because they have an unconditional preference for fairness but because subjects want to be seen as fair. Thus, subjects reduce giving in conditions of high anonymity (Dana / Weber / Kuang 2007; Hoffman / McCabe / Smith 1996; Andreoni / Bernheim 2009; Franzen / Pointner 2012). Similarly, subjects show more pro-social behavior if they feel observed. Evidence from lab and field experiments suggests that subtle cues such as „watching eyes“ are sufficient to elicit more generous behavior (Bateson / Nettle / Roberts 2006; Ekström 2012; Ernest-Jones / Nettle / Bateson 2011; Francey / Bergmüller 2012; Haley / Fessler 2005; Rigdon et al. 2009). If subtle cues are able to induce more generous behavior then the mere presence of others in a group could be sufficient to do the same. Hence, individuals in groups may feel observed by the other decision-makers, and such an observation effect may increase their motivation to behave in a socially desirable way, e.g. to be more generous. ${ }^{3}$

On the other side, actors are relieved from exclusive responsibility of their decisions in groups since group members can ascribe the violation of norms to the group. This effect is termed „diffusion of responsibility“ and numerous experiments have demonstrated its existence (e.g. Darley / Latané 1968; Freeman et al. 1975). Also, experiments with the public goods game have shown that the free rider problem becomes more severe with increasing group size (Marwell / Ames 1979; but see also Isaac / Walker 1988, 1994 for different results).

Moreover, the psychological literature suggests that aspects of the discussion process of groups can influence the outcome (Schulz-Hardt / Brodbeck 2012). Particularly, Tversky and Kahneman (1974, see also Kahneman 1992) demonstrated that „anchors“ can cause a cognitive bias and influence the decision-making. Anchors may have consequences even though the provided information is irrelevant or exaggerated (e.g. Wilson et al. 1996). Presumably, anchors are influential because they induce a cognitive selection process. Hence, subjects perceive information confirming the anchor to be more plausible. Similarly, in the group dictator game the first suggestion by a group member may serve as an anchor and

3 The effect is also called, image scoring“ by Nowak and Sigmund (1998). Players try to build up a positive reputation or an ,image score" in order to gain benefits in future interaction (Wedekind / Milinski 2000). 
may influence the group more than later arguments. Besides a cognitive influence the first suggestion might additionally have a normative effect. The first proposal provides a hint to members of what might be a socially desirable choice. Therefore, the first suggestion might also be important because it sets a moral standard which influences the direction of the group discussion.

Summing up, there are four partly contradicting and partly complementing hypotheses specifying the possible difference between single actors and group decisions in the dictator game: (1) Since a group dictator game does not involve a cognitive or strategic problem the possibility of discussing the distribution does not facilitate the choice. Hence, a group's outcome should simply be determined by the group's composition, e.g. the preference of its members. If a majority consists of selfish players, the group's distribution should be selfish, while a majority of generous players should translate into generous group decisions. (2) Groups might behave to maximize payoff more than individuals would because groups enhance competitiveness and induce a diffusion-of-responsibility effect. Both effects can lead groups to allocate less of their endowment to others as compared to individuals. (3) Groups might be more altruistic than individuals because of the observer effect. (4) A group's decision depends on aspects of the discussion process. Particularly, the first suggestion made by a group member could serve as an anchor and influences the group's final outcome.

So far, there are only two studies investigating the difference between individual and group decision-making in the dictator game. Cason and Mui (1997) conducted an experiment in which individuals were asked to distribute an endowment of $\$ 5$ first individually and later in groups of two players. ${ }^{4}$ They find that the dyads, which discussed the decision face-to-face, gave on average $27.6 \%$ of the endowment as compared to individuals who gave $26.1 \% .^{5}$ Thus, groups are slightly more other-regarding than are individuals. Luhan et al. (2009) conduct three-person group dictator games. First, individuals played the standard dictator game and received $\$ 5$ endowment for allocation. Second, groups of three players were matched systematically according to whether individuals gave nothing or little, moderate amounts, or larger amounts in the first round. They find that groups donate less to others than do individuals ( $\$ 0.94$ or $19 \%$ versus $\$ 0.54$ or $11 \%$ ) and that this selfish shift is driven by the preference of the most selfish player in the group. Luhan et al. (2009) explain the difference of their finding to the Cason and Mui (1997) study basically by the observer effect. In the former study subjects communicated face-to-face while in the latter they communicated via instant messages through a chat program, preserving individuals' anonymity.

In what follows, we replicate the study of Luhan et al. (2009) and extend it by incorporating a more detailed analysis of the chat protocols in the group condition. Furthermore, we match individuals randomly into groups and not systematically as in Luhan et al. (2009). Random assignment introduces more variation between groups as compared to assigning to each group a selfish, moderate and altruistic player. Hence, we are able to assess the effect of group composition on group giving. The next section describes our experimental design in detail and also points out the differences to the Luhan et al. (2009) study.

4 This sequence was also reversed.

5 We calculated these relative offers on the basis of data appended in Table A1 and A2 in Cason and Mui (1997) (weighted means are used). The difference between individual and team offers is statistically not significant (paired two-sided t-test, $t=-0.73, p=0.47$ ). However, Cason and Mui (1997) interpret their results as an altruistic shift in groups and explain this shift by social comparison theory, which states that individuals like to be more other-regarding than the average. 


\section{Experimental procedure}

In our experiment subjects first played a standard single dictator game (double-blind). This first stage served to identify the social preferences of participants. Second, the same subjects were randomly matched into groups of three and participated in a group dictator game (see Figure 1$){ }^{6}$

Participants were recruited from the student population of the University of Cologne. ${ }^{7}$ In all, 114 subjects showed up for the experiment. Since we needed a number divisible by three in each session we had to send some participants home. Those received a show-up fee of $€ 5$. Therefore, we ended up with 90 subjects, 65 females and 25 males who were on average 22.4 years old.

Upon arrival at the lab, subjects drew an ID number and selected the corresponding working place. The laboratory was equipped with 18 booths, each with a PC that displayed the instructions. ${ }^{8}$ Generally, each session had 9-15 participants. The experiment started with the single dictator game (SDG) as soon as all subjects had arrived in the laboratory. In the SDG each participant received $€ 10$, which they could distribute any way they wished between themselves and another student of the university. All subjects were proposers and could only contribute integer amounts. The recipients were not present in the room and did not participate in the experiment, but were randomly chosen from the student population and received the money after the experiment. After completion of the first stage, the subjects were randomly matched into groups of three, each without knowing the identity of the other two group members. The groups received an endowment of $€ 30$ which they had to distribute in any way they wanted between their own group and another group of three subjects. The group was told that whatever amount they kept would be divided equally among the members of their own group.

In the GDG, the three group members were able to communicate via instant messaging. Participants were told that the group had three minutes' time to come up with a decision on which amount to donate to the other group. If the group did not make a decision within three minutes, they were given another three minutes, but were told that they would receive nothing if no decision was reached after the second round. There are two possibilities for how groups can democratically reach a decision: through unanimity or majority vote. We implemented both (randomly determined) to control for possible effects on the decision outcome.

After participating in both the single and the group dictator games, participants completed an online questionnaire to collect socio-demographic information. As soon as subjects had completed the questionnaire, they left the laboratory and went to the payout room where they picked up an envelope containing their combined earnings from the SDG and GDG. Subjects picked the envelope by the ID number they had drawn earlier. The payout was completely anonymous. There was no face-to-face contact between subjects and the experimenter or any other persons in the payout room. The money donated to others from the single or group dictator games was later allocated to randomly chosen students of the university.

6 In Luhan et al. (2009) subjects also played a further single dictator game after the group condition in order to control for learning effects. They also conducted a control group in which individual subjects played only the single dictator game three consecutive times. Since the results did not show learning effects we decided to drop the third stage.

7 Student volunteers were recruited on campus, but did not come from a managed subject pool.

8 We used z-tree (Fischbacher 2007). Instructions for the participants are available upon request by the authors. 
Figure 1: The SDG-GDG-design
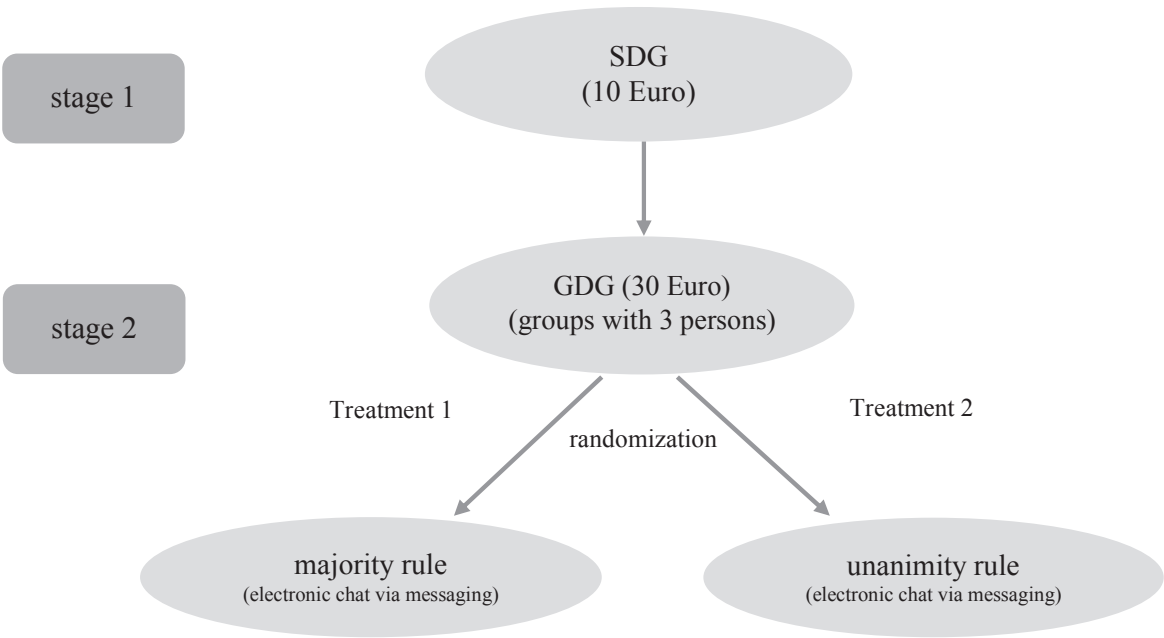

\section{Results}

The results are displayed in Figure 2 and show the distribution of the amounts allocated by the 90 subjects in the SDG. On average, subjects donated $€ 2.36$ to their co-players. Thirtyeight subjects (42\%) gave nothing, $24(27 \%)$ allocated moderate amounts between $€ 1$ and $€ 4$, and 28 participants (31\%) donated half or more to co-players.

After completing one round of the SDG, groups of three players were matched randomly by the computer program. Subjects did not know who their co-players in the group were. Matching the groups randomly resulted in various compositions of the 30 groups. Twelve groups had a majority of selfish subjects who allocated nothing to their co-players in the SDG. Seventeen groups had a majority of players who gave either moderate amounts or half, and one group consisted of three players who all gave half or more in the SDG. None of our groups consisted exclusively of selfish subjects. ${ }^{9}$ With regard to gender composition, we ended up with 1 completely male group (MMM), while 4 groups had a male majority (MMF), 14 groups had a female majority (FFM), and 11 groups were completely female (FFF). Fourteen groups were randomly assigned to the majority treatment and 16 to the unanimity condition. All groups reached a decision within the given time. The fastest group agreed within 37 seconds on how to make the distribution and the slowest group took 290 seconds. On average groups agreed within 106 seconds on a decision. The amounts given to the other groups in the GDG are displayed in Figure 3.

9 We also tested other classifications of other-regarding giving, e.g. interpreting the giving of up to two euro as selfish behavior. This variation did not influence our results. 
Figure 2: Amount given to co-player in the SDG $(\bar{x}=2.36, N=90)$

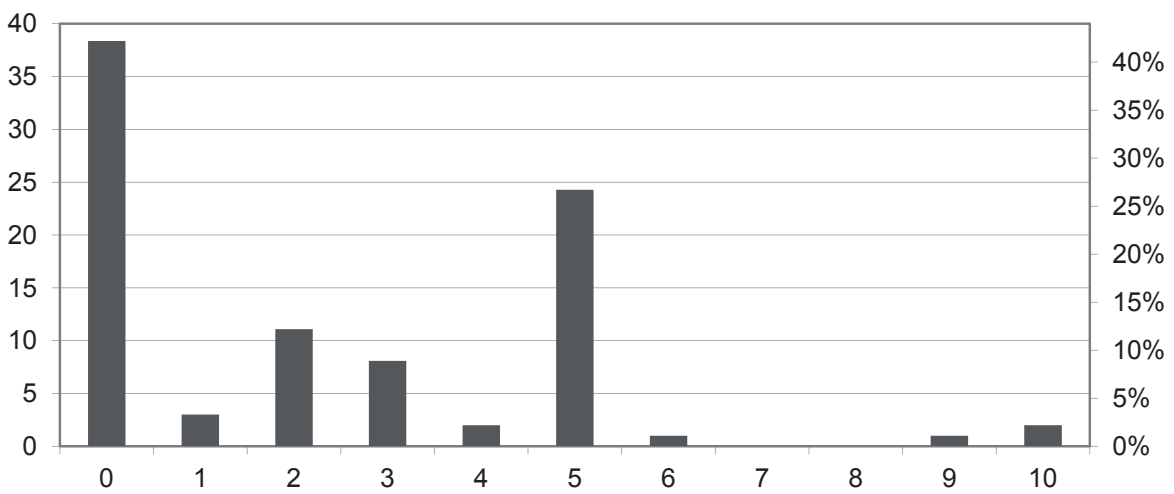

Figure 3: Amount given to recipients in the GDG $(\bar{X}=7.27$, per subject $\bar{X}=2.42 ; N=30)$

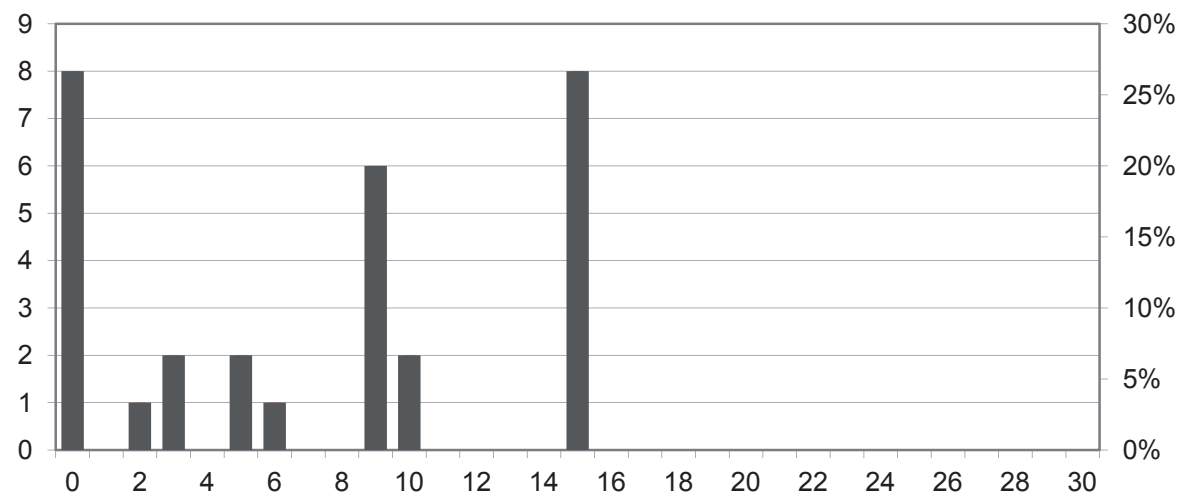

The distribution obtained is bimodal. Eight groups donated nothing and another eight groups gave away exactly half of their endowment. The other 14 groups gave some amount in between. The proportion of groups that gave half of their endowment is $26.7 \%$, which is very close to the proportion of individuals who gave away half in the SDG. The proportion that gave nothing decreased somewhat in the GDG when compared to the proportion of individuals giving nothing in the SDG. However, the average contribution of groups in the GDG is $€ 7.27$, which results in $€ 2.42$ per group member. This is not significantly different from the $€ 2.36$ given in the SDG (paired t-test, $t=0.173, p=0.864$ ). This result means that groups become neither more rational nor more altruistic in the dictator game as compared to individuals. Thus, the results support hypothesis 1 and reject the conclusion of Luhan et al. (2009) as well as the finding by Cason and Mui (1997).

Next, we analyze the determinants of the groups' allocation. First, we regress only the groups' composition, determined by the allocation in the first stage, on the groups' allocation. One way of doing this is to classify groups according to the number of selfish, moderate, and fair players. Since we have only one group with solely fair players, and none with exclusively selfish players, we dichotomized groups in those with a majority of selfish players and fair and moderate players. The results of this OLS-regression are displayed in col- 
umn 1 of Table $1 .{ }^{10}$ The results reveal that the group composition drives the amount donated to others in the group. Groups that have a majority of selfish players donate on average $€ 4.4$ less than groups with a majority of fair or moderate players. Another way of taking the composition into account would be to add the individual allocation of stage one and regress it on the groups' allocation. The result is basically the same. For every euro given in round one by the group members individually, groups give $€ 0.66$ more.

Table 1: OLS (models 1-3) and Tobit (4-6) estimates, dependent variable is amount given by group

\begin{tabular}{|c|c|c|c|c|c|c|}
\hline & (1) & (2) & (3) & (4) & (5) & (6) \\
\hline preference composition of & -4.35 & 1.49 & 1.48 & -6.71 & 2.03 & 1.88 \\
\hline group $(1=$ majority egoistic $)$ & $(2.21)$ & $(1.08)$ & $(1.05)$ & $(3.45)$ & $(1.31)$ & $(1.16)$ \\
\hline \multirow[t]{2}{*}{ decision rule (1=unanimity) } & & 2.14 & 2.11 & & 2.27 & $2.47^{*}$ \\
\hline & & $(1.21)$ & $(1.17)$ & & $(1.34)$ & $(1.13)$ \\
\hline \multirow[t]{2}{*}{ number of selfisch arguments } & & $-2.32^{* * *}$ & $-1.72^{* * *}$ & & $-3.20^{* * * *}$ & $-2.54^{* * * *}$ \\
\hline & & $(0.23)$ & $(0.39)$ & & $(0.36)$ & $(0.45)$ \\
\hline \multirow[t]{2}{*}{ number of moderate arguments } & & 0.14 & $0.42^{*}$ & & $0.56^{*}$ & $0.87^{* * *}$ \\
\hline & & $(0.18)$ & $(0.19)$ & & $(0.23)$ & $(0.28)$ \\
\hline number of equal split and large & & $1.79^{* * * *}$ & $1.48^{* * * *}$ & & $2.30^{* * * *}$ & $1.95^{* * *}$ \\
\hline arguments & & $(0.23)$ & $(0.22)$ & & $(0.34)$ & $(0.29)$ \\
\hline \multirow[t]{2}{*}{ group with majority male $(=1)$} & & & 0.174 & & & -0.48 \\
\hline & & & $(1.65)$ & & & $(1.90)$ \\
\hline group with majoritiy female $(=1)$ & & & 0.67 & & & 1.38 \\
\hline (reference comletely female & & & $(1.42)$ & & & $(1.50)$ \\
\hline \multicolumn{7}{|l|}{ groups) } \\
\hline \multirow[t]{2}{*}{ first suggestion in discussion } & & & $0.32 *$ & & & $0.30 *$ \\
\hline & & & $(0.13)$ & & & $(0.13)$ \\
\hline \multirow[t]{2}{*}{ Constant } & $8.57^{* * * *}$ & $6.30^{* * * *}$ & 3.23 & $8.07^{* * *}$ & $4.59^{*}$ & 1.34 \\
\hline & $(1.25)$ & $(1.53)$ & (2.17) & $(1.503)$ & (1.74) & $(2.07)$ \\
\hline Ovservations & 30 & 30 & 30 & 30 & 30 & 30 \\
\hline Adjusted $R^{2}$ & 0.087 & 0.763 & 0.787 & & & \\
\hline Log-Likelihood & & & & -81.39 & -57.43 & -55.12 \\
\hline
\end{tabular}

Standard errors in parentheses, ${ }^{*} p<0.05,{ }^{* *} p<0.01,{ }^{* * *} p<0.001$

Next, we analyze the chat protocols of the groups. ${ }^{11}$ Overall, the groups exchanged 2086 words. Word frequency is pretty evenly distributed between selfish, moderate, and fair players. A typical selfish proposal is formulated something like „I think we should keep everything“. Seventy-eight percent of all selfish proposals were written by selfish players $(n=56$, see Table 2). A moderate argument usually states „We should give a small amount“. Such arguments $(n=67)$ were usually also made by selfish $(42 \%)$ or moderate players $(33 \%)$. Finally we also find equal split (e.g. ,50:50?“) arguments $(n=57)$, which were mostly formulated by fair players $(58 \%)$. Thus, players predominantely made suggestions in the group that corresponded to their preferences revealed in the first stage. This conclusion is confirmed by a chi-square test of table $2\left(\chi^{2}=50.27, p=0.000\right)$.

10 In addition, we estimated Tobit-regressions to account for the left-censored dependent variable. Since the results remain robust we interpret only OLS-coefficients. For the descriptive statistics see also Table A in the Appendix.

11 An example of such a discussion protocol is given in the Appendix (Table B). Classifications of the arguments in the chats were done by both authors, first independently and later by clarifying discrepant classifications. 
Table 2: The association of player type and arguments in group discussion

\begin{tabular}{|c|c|c|c|c|}
\hline & selfish player & moderate player & $\begin{array}{c}\text { other-regarding } \\
\text { player }\end{array}$ & \\
\hline selfish proposal* & $\begin{array}{c}44 \\
78.6 \% \\
(52.4 \%)\end{array}$ & $\begin{array}{c}8 \\
14.3 \% \\
(19.0 \%)\end{array}$ & $\begin{array}{c}4 \\
7.1 \% \\
(7.4 \%)\end{array}$ & $\begin{array}{c}56 \\
100.0 \% \\
(31.1 \%)\end{array}$ \\
\hline moderate proposal* & $\begin{array}{c}28 \\
41.8 \% \\
(33.3 \%)\end{array}$ & $\begin{array}{c}22 \\
32.8 \% \\
(52.4 \%)\end{array}$ & $\begin{array}{c}17 \\
25.4 \% \\
(31.5 \%)\end{array}$ & $\begin{array}{c}67 \\
100.0 \% \\
(37.2 \%)\end{array}$ \\
\hline equal split proposal* & $\begin{array}{c}12 \\
21.05 \% \\
(14.3 \%)\end{array}$ & $\begin{array}{c}12 \\
21.05 \% \\
(28.6 \%)\end{array}$ & $\begin{array}{c}33 \\
57.9 \% \\
(61.1 \%)\end{array}$ & $\begin{array}{c}57 \\
100.0 \% \\
(31.7 \%)\end{array}$ \\
\hline & $\begin{array}{c}84 \\
46.7 \% \\
(100.0 \%)\end{array}$ & $\begin{array}{c}42 \\
23.3 \% \\
(100.0 \%)\end{array}$ & $\begin{array}{c}54 \\
30.0 \% \\
(100.0 \%)\end{array}$ & $\begin{array}{c}180 \\
100.0 \% \\
(100.0 \%)\end{array}$ \\
\hline
\end{tabular}

* Absolute number of arguments in discussion; column percentages in parentheses; $\chi^{2}=50.27(\mathrm{df}=4$, $p<0.001$ ), Cramér's $V=0.37$

Next we introduce the number of selfish, moderate, and fair arguments into the regression (see Table 1, model 2). The results indicate that the amount allocated by groups depends on the number of selfish, moderate, and fair arguments exchanged in the group. As expected, more selfish arguments led groups to give less, while moderate and equal split arguments induced groups to give more. ${ }^{12}$ Finally, we introduce further control variables into the regression equation (Table 1, model 3). The results show that the gender composition does not influence the groups' allocations. The decision rule (unanimity versus majority) is only significant at the $10 \%$ level and can probably not be generalized. Since we have no convincing theoretical argument of why unanimity should matter, we suggest not to over-interpret this result. Model 3 of Table 1 also includes the first suggested offer in the analysis. The result shows that higher first suggestions influence the group to donate more endowments to the other group. Therefore, this result confirms our hypothesis that the first suggestion made by a group member serves as an anchor.

Taking only the preference composition into account explains only nine percent of the variance of the groups' allocation. Including the number of arguments as well as the level of the first suggestion increases the explained variance to $79 \%$. Thus, we are rather confident that the variables taken into consideration capture the essential aspects of the decision processes in the GDG.

\section{Conclusion and discussion}

Former studies comparing groups' allocations to individual donations in the dictator game come up with inconclusive evidence. Cason and Mui (1997) suggest that groups are more generous in the dictator game. They explain the altruistic shift of groups by social comparison theory, which states that individuals in groups are motivated to be more other-regarding. Luhan et al. (2009) find that groups give less than individuals in the dictator game. Their central result is that group giving is determined by the preference of the most selfish group member. Hence, groups shift towards lower allocations. However, our replication study does not confirm either result. We find that groups give according to the arguments made in the

12 The two coefficients $\beta=-1.72$ (for the number of selfish arguments) and $\beta=1.48$ (for the number of equal split and large arguments) are equal in strength and statistically not different $(F=3.71$, $p=0.068)$. Hence, selfish arguments and equal split arguments influence the groups' allocations in a similar way. 
chats (see Table 1) and that the type of the proposal is determined by players' preferences as revealed in the SDG. ${ }^{13}$ Thus, group members transfer their preference into groups by making proposals consistent with their choice in the single dictator game. Furthermore, our analysis demonstrates that the number of selfish, moderate or other-regarding arguments exchanged during the group discussion drives the final group allocation. Selfish arguments influence the group to give less, while moderate or fair arguments drive the group to donate more. Hence, there is no mystery involved in group decision-making and the decisions of groups are explained by the members' preferences.

We believe that our result is theoretically more plausible than the findings of the two former studies. The results of Cason and Mui (1997) can most likely be explained by an observation effect since their two-person groups involved face-to-face communication and therefore have lower anonymity than the individual decision situation. Moreover, the results by Cason and Mui (1997) are not decisive as the means of groups' and individuals' allocations does not differ significantly. Hence, their results can also be interpreted like our results that there are no differences between decisions of groups and individuals.

The results of Luhan et al. (2009) could be an artifact of their design. The authors matched players systematically by composing the groups so that they consist of ,relatively“ selfish, moderate or other-regarding players. Therefore, in some groups „selfish“ players gave moderate amounts. Hence, moderate players also drive the groups' decisions. It is hard to see why the relative preference of players should determine the outcome, particularly since players did not know their respective behavior from the first stage and therefore had no information about their relative position.

Our study also presents some further results that were not addressed by Cason and Mui (1997) or Luhan et al (2009). Thus, we were not able to replicate the finding of Dufwenberg and Muren (2006) that female groups are more generous than male groups. ${ }^{14}$ Furthermore, we find that the first suggestion made in a group has an additional influence on the group out-come. This finding can be explained by the anchor effect proposed by Tversky and Kahneman (1974). The first suggestion provides a starting point and orientation for the group on what to do. This result does not mean that the group follows the first suggestion, but it indicates that the first proposal is influential. However generalizing the finding to real groups is premature since it is obtained in a laboratory under very special conditions. In real life most groups or committees are certainly not involved in one-shot anonymous types of decisionmaking.

Our study also has some shortcomings, which could have influenced the results. First, we restricted the decision time to six minutes (two rounds of three minutes). Even though most groups used much less time (106 seconds on average) this constraint may have created the impression that decision time is an issue. Future research should try not to limit the decision time and to see whether the impact of the first suggestion is still dominant. Second, our experiment involved only three-person groups, as did the studies with GDG in the past. However, most decision groups in real life are bigger and the question is left open as to whether discussion and decision processes differ in larger groups.

13 This result is also confirmed by OLS-regressions, in which the number of selfish arguments (1) or the number of other-regarding arguments (2) are the dependent variables. The result is shown in the appendix (Table C).

14 Contrary to Dufwenberg and Muren (2006), in our experiment the gender of the group members was not visible. Dufwenberg and Muren (2006) used face-to-face interactions which could have caused the divergent result. 


\section{References}

Andreoni, J. / B.D. Bernheim (2009): Social Image and the 50-50 Norm: A Theoretical and Experimental Analysis of Audience Effects, in: Econometrica 77, S. 1607-1636.

Bateson, M. / D. Nettle/ G. Roberts (2006): Cues of Being Watched Enhance Cooperation in a RealWorld Setting, in: Biology Letters 2, S. 412-414.

Blinder, A.S. / J. Morgan (2005): Are Two Heads Better than One? Monetary Policy by Committee, in: Journal of Money, Credit and Banking 37, S. 789-811.

Bornstein, G. / T. Kugler / A. Ziegelmeyer (2004): Individual and Group Decisions in the Centipede Game: Are Groups More "Rational" Players?, in: Journal of Experimental Social Psychology 40, S. 599-605.

Camerer, C. (2003): Behavioral Game Theory: Experiments in Strategic Interaction, New York / NY.

Cason, T. N. / V. L. Mui (1997): A Laboratory Study of Group Polarization in the Team Dictator Game, in: The Economic Journal 107, S. 1465-1483.

Charness, G. / M. Sutter (2012): Groups Make Better Self-Interested Decisions, in: Journal of Economic Perspective 26, S. 157-176.

Charness, G. / E. Karni / D. Levin (2007): Individual and Group Decision Making under Risk: An Experimental Study of Bayesian Updating and Violations of First-Order Stochastic Dominance, in: Journal of Risk and Uncertainty 35, S. 129-148.

Charness, G. / L. Rigotti / A. Rustichini (2007): Individual Behavior and Group Membership, in: American Economic Review 97, S. 1340-1352.

Dana, J. / R.A. Weber / J.X. Kuang (2007): Exploiting Moral Wiggle Room: Experiments Demonstrating an Illusory Preference for Fairness, in: Economic Theory 33, S. 67-80.

Darley, J.M. / B. Latané (1968): Bystander Intervention in Emergencies: Diffusion of Responsibility, in: Journal of Personality and Social Psychology 8, S. 377-383.

Dufwenberg, M. / A. Muren (2006): Gender Composition in Teams, in: Journal of Economic Behavior and Organization 61, S. 50-54.

Ekström, M. (2012): Do Watching Eyes Affect Charitable Giving? Evidence from a Field Experiment, in: Experimental Economics 15, S. 530-546.

Engel, C. (2011): Dictator Games: A Meta Study, in: Experimental Economics 14, S. 583-610.

Ernest-Jones, M. / D. Nettle / M. Bateson (2011): Effects of Eye Images on Everyday Cooperative Behavior: A Field Experiment, in: Evolution and Human Behavior 32, S. 172-178.

Fischbacher, U. (2007): z-Tree: Zurich Toolbox for Ready-Made Economic Experiments, in: Experimental Economics 10, S. 171-178.

Francey, D. / R. Bergmüller (2012): Images of Eyes Enhance Investments in a Real-Life Public Good, in: PLoS ONE 7, e37397.

Franzen, A. / S. Pointner (2012): Anonymity in the Dictator Game Revisited, in: Journal of Economic Behavior and Organization 81, S. 74-81.

Freeman, S. / M.R. Walker / R. Borden / B. Latané (1975): Diffusion of Responsibility and Restaurant Tipping: Cheaper by the Bunch, in: Personality and Social Psychology Bulletin 1, S. 584-587.

Gillet, J. / A. Schram / J. Sonnemans (2009): The Tragedy of the Commons Revisited: The Importance of Group Decision-Making, in: Journal of Public Economics 93, S. 785-797.

Haley, K.J. / D.M.T. Fessler (2005): Nobody's Watching? Subtle Cues Affect Generosity in an Anonymous Economic Game, in: Evolution and Human Behavior 26, S. 245-256.

Hoffman, E. / K. McCabe / V. Smith (1996): Social Distance and Other-Regarding Behavior in Dictator Games, in: American Economic Review 86, S. 653-660. 
Insko, C.A. / J. Schopler / R.H. Hoyle / G.J. Dardis / K.A. Graetz (1990): Individual-Group Discontinuity as a Function of Fear and Greed, in: Journal of Personality and Social Psychology 58, S. 68-79.

Isaac, M.R. / J.M. Walker (1988): Group Size Effects in Public Goods Provision: The Voluntary Contribution Mechanism, in: Quarterly Journal of Economics 103, S. 179-200.

Isaac, M.R. / J.M. Walker (1994): Group Size and the Voluntary Provision of Public Goods: Experimental Evidence Utilizing Large Groups, in: Journal of Public Economics 54, S. 1-36.

Kahneman, D. / J.L. Knetsch / R. Thaler (1986): Fairness and the Assumptions of Economics, in: Journal of Business 59, S. 285-300.

Kahneman, D. (1992): Reference Points, Anchors, Norms, and Mixed Feelings, in: Organizational Behavior and Human Decision Processes 51, S. 296-312.

Kocher, M. / M. Sutter (2005): The Decision Maker Matters: Individual versus Group Behavior in Experimental Beauty-Contest Games, in: The Economic Journal 115, S. 200-23.

Kocher, M. / M. Sutter (2007): Individual Versus Group Behavior and The Role Of The Decision Making Procedure in Gift-Exchange Experiments, in: Empirica 34, S. 63-88.

Kugler, T. / G. Bornstein / M.G. Kocher / M. Sutter (2007): Trust Between Individuals and Groups: Groups Are Less Trusting Than Individuals But Just as Trustworthy, in: Journal of Economic Psychology 28, S. 646-657.

Larson, J.R. (2010): In Search of Synergy in Small Group Performance, New York / NY.

Laughlin, P.R. / B.L. Bonner / A.G. Miner (2002): Groups Perform Better Than The Best Individuals On Letters-to-Numbers Problems, in: Organizational Behavior and Human Decision Processes 88, S. 605-20.

Luhan, W.J. / M.G. Kocher / M. Sutter (2009): Group Polarization in The Team Dictator Game Reconsidered, in: Experimental Economics 12, S. 26-41.

Marwell, G. / R.E. Ames (1979): Experiments On The Provision of Public Goods. I. Resources, Interest, Group Size, and the Free-Rider-Problem, in: American Journal of Sociology 84, S. 1335-1360.

Nowak, M.A. / K. Sigmund (1998): The Dynamics of Indirect Reciprocity, in: Journal of Theoretical Biology 194, S. 561-574.

Rigdon, M. / K. Ishii / M. Watabe / S. Kitayama (2009): Minimal Social Cues in the Dictator Game, in: Journal of Economic Psychology 30, S. 358-367.

Schulz-Hardt, S. / F.C. Brodbeck (2012): Group Performance and Leadership, in: M. Hewstone, M. / W. Stroebe / K. Jonas, K. (eds.), An Introduction to Social Psychology, Glasgow, S. 415-448.

Sutter, M. (2005): Are Four Heads Better Than Two? An Experimental Beauty-Contest Game With Teams of Different Size, in: Economics Letters 88, S. 41-46.

Sutter, M. / M.G. Kocher / S. Strauss (2009): Individuals and Teams in Auctions. Oxford Economic Papers-New Series 61, S. 380-394.

Tajfel, H. (1978): Social Categorization, Social Identity and Social Comparison, in: H. Tajfel (ed.), Differentiation between Social Groups: Studies in the Social Psychology of Intergroup Relations, London, S. 61-76.

Tversky, A. / D. Kahneman (1974): Judgment Under Uncertainty: Heuristics and Biases, in: Science New Series 185, S. 1124-1131.

Wedekind, C. / M. Milinski (2000): Cooperation Through Image Scoring in Humans, in: Science New Series 288, S. 850-852.

Wildschut, T. / B. Pinter / J.L. Vevea / C.A. Insko / J. Schopler (2003): Beyond the Group Mind: A Quantitative Review of the Interindividual-Intergroup Discontinuity Effect, in: Psychological Bulletin 129, S. 698-722.

Wilson, T.D. / C.E. Houston / K.M. Etling / N. Brekke (1996): A New Look at Anchoring Effects: Basic Anchoring and Its Antecedents, in: Journal of Experimental Psychology: General 125, S. 387-402. 
Prof. Dr. Axel Franzen

Universität Bern Institut für Soziologie

Fabrikstr. 8

3012 Bern

franzen@soz.unibe.ch

Prof. Dr. Sonja Pointner Ludwig-Maximilians-Universität München Institut für Soziologie

Konradstr. 6

80801 München

sonja.pointner@soziologie.uni-muenchen.de

\section{Appendix}

Table A: Descriptive statistics

\begin{tabular}{|c|c|c|c|c|c|}
\hline & Mean & SD & Min & Max & $N$ \\
\hline $\begin{array}{l}\text { preference composition of group } \\
\text { (1= majority egoistic) }\end{array}$ & 0.3 & 0.47 & 0 & 1 & 30 \\
\hline $\begin{array}{l}\text { sum of individual allocations } \\
\text { (in stage 1) of group members }\end{array}$ & 7.07 & 3.31 & 2 & 15 & 30 \\
\hline $\begin{array}{l}\text { majority female or complete female group } \\
\text { (coded 1) }\end{array}$ & 0.83 & 0.38 & 0 & 1 & 30 \\
\hline group with majority male subjects (coded 1$)$ & 0.17 & 0.38 & 0 & 1 & 30 \\
\hline only female subjects in group (coded 1) & 0.47 & 0.51 & 0 & 1 & 30 \\
\hline number of selfish arguments in group & 1.87 & 2.03 & 0 & 7 & 30 \\
\hline number of moderate arguments in group & 2.23 & 2.27 & 0 & 8 & 30 \\
\hline $\begin{array}{l}\text { number of equal split (or larger) arguments in } \\
\text { group }\end{array}$ & 1.9 & 2.23 & 0 & 9 & 30 \\
\hline first suggestion (for allocation to other group) & 4.97 & 6.23 & 0 & 20 & 30 \\
\hline $\begin{array}{l}\text { decision rule: unanimity versus majority rule } \\
(1=\text { unanimity) }\end{array}$ & 0.53 & 0.51 & 0 & 1 & 30 \\
\hline number of words in group discussion & 69.53 & 50.71 & 19 & 236 & 30 \\
\hline
\end{tabular}


Table B: Example of chat protocol (translated from German)

\begin{tabular}{|c|c|c|c|}
\hline chatname & person & message & time $(\mathrm{sec})$ \\
\hline 999 & F1 & Keep everything? & 10 \\
\hline 742 & M1 & Morning. I would give $50 / 50$ & 11 \\
\hline 728 & F2 & I would share 15 & 16 \\
\hline 999 & F1 & I'd like to keep everything & 22 \\
\hline 728 & F2 & 15 & 32 \\
\hline 999 & F1 & Giving something is not meaningful & 34 \\
\hline 999 & F1 & Why do you want to give something? & 41 \\
\hline 742 & M1 & Because we are nice people;) & 47 \\
\hline 728 & F2 & Then everybody earns something & 51 \\
\hline 999 & F1 & But with more money you can afford more. & 58 \\
\hline 742 & M1 & $\begin{array}{l}\text { And if the others behave similar we also get } 30 \text { al- } \\
\text { together }\end{array}$ & 62 \\
\hline 999 & F1 & But the others will not particpate... & 74 \\
\hline 742 & M1 & But that's society & 78 \\
\hline 742 & M1 & No trust & 80 \\
\hline 999 & F1 & No. I understood that in another way & 85 \\
\hline 728 & $\mathrm{~F} 2$ & Hm, why not $20 ?$ & 105 \\
\hline 999 & F1 & $\begin{array}{l}\text { Some other students were chosen, not somebody } \\
\text { from the room }\end{array}$ & 106 \\
\hline 742 & M1 & They don't get the $€ 15$ ? & 112 \\
\hline 999 & F1 & 25 would be okay & 113 \\
\hline 728 & $\mathrm{~F} 2$ & That doesn't matter & 119 \\
\hline 999 & F1 & That means that you get nothing for giving & 135 \\
\hline 742 & M1 & $\begin{array}{l}\text { I think keeping everything is not ok. Because this } \\
\text { leads to a societal dilemma }\end{array}$ & 142 \\
\hline 728 & F2 & And now? & 148 \\
\hline 728 & F2 & Only 30 seconds left & 153 \\
\hline 742 & M1 & $20 ?$ & 154 \\
\hline 728 & F2 & Ok $20 ?$ & 160 \\
\hline 728 & F2 & Yes 20 & 170 \\
\hline 999 & F1 & Would be for 30 , but ok & 170 \\
\hline 728 & F2 & Ok, copy that & 177 \\
\hline 742 & M1 & Let`s make 21 & 178 \\
\hline 728 & $\mathrm{~F} 2$ & 20 & 178 \\
\hline
\end{tabular}

Table C: OLS estimates, dependent variables are the number of selfish (model 1) and the number of moderate, equal split, and large arguments (model 2)

\begin{tabular}{lcc}
\hline & $(1)$ & $(2)$ \\
\hline $\begin{array}{l}\text { preference composition of } \\
\text { group (1= majority egoistic) }\end{array}$ & $1.64^{*}$ & $1.68^{*}$ \\
& $(0.73)$ & $(0.74)$ \\
gender composition of group & & \\
(1= majority female or & 0.70 & 1.33 \\
complete female group) & $(0.49)$ & $(1.05)$ \\
number of words in group & & \\
discussion & $0.02^{* * *}$ & $0.05^{* * *}$ \\
constant & $(0.01)$ & $(0.01)$ \\
& -0.59 & -0.13 \\
Observations & $(0.61)$ & $(1.16)$ \\
Adjusted $R^{2}$ & 30 & 30 \\
\hline
\end{tabular}

